\title{
Cordycepin inhibits chondrocyte hypertrophy of mesenchymal stem cells through PI3K/Bapx1 and Notch signaling pathway
}

\author{
Zhen Cao ${ }^{1,2}$, Ce Dou ${ }^{2}$, Jianmei $\mathrm{Li}^{2}$, Xiangyu Tang ${ }^{2}$, Junyu Xiang ${ }^{2}$, Chunrong Zhao ${ }^{2}$, Lingyu Zhu', Yun Bai ${ }^{2}$, \\ Qiang Xiang ${ }^{3}$ \& Shiwu Dong, \\ ${ }^{1}$ Department of Anatomy, Third Military Medical University, ${ }^{2}$ National \& Regional United Engineering Laboratory of Tissue Engineering, \\ Department of Biomedical Materials Science, School of Biomedical Engineering, Third Military Medical University, ${ }^{3}$ Department of \\ Emergency, Southwest Hospital, Third Military Medical University, Chongqing 400038, China
}

\begin{abstract}
Mesenchymal stem cells (MSCs) are widely used in cartilage tissue engineering to repair articular cartilage defects. However, hypertrophy of chondrocytes derived from MSCs might hinder the stabilization of hyaline cartilage. Thus, it is very important to find a suitable way to maintain the chondrogenic phenotype of chondrocytes. It has been reported that cordycepin has anti-inflammatory and anti-tumor functions. However, the role of cordycepin in chondrocyte hypertrophy remains unclear. Therefore, the objective of this study was to determine the effect of cordycepin on chondrogenesis and chondrocyte hypertrophy in MSCs and ATDC5 cells. Cordycepin upregulated chondrogenic markers including Sox9 and collagen type II while down-regulated hypertrophic markers including Runx2 and collagen type $X$. Further exploration showed that cordycepin promoted chondrogenesis through inhibiting Nrf2 while activating BMP signaling. Besides, cordycepin suppressed chondrocyte hypertrophy through PI3K/Bapx1 pathway and Notch signaling. Our results indicated cordycepin had the potential to maintain chondrocyte phenotype and reconstruct engineered cartilage. [BMB Reports 2016; 49(10): 548-553]
\end{abstract}

\section{INTRODUCTION}

Acute cartilage defects may be caused by a comminuted or displaced intra-articular fracture. However, a chronic articular cartilage defect is often caused by matrix degradation during the progression of osteoarthritis (1-3). Limited by the intrinsic repairing capacity of cartilage tissue, repairing damaged articular cartilage currently faces major obstacles (4). Tissue

*Corresponding author. Tel: +86-2368771270; Fax: +862368771270; E-mail: dongshiwu@tmmu.edu.cn

https://doi.org/10.5483/BMBRep.2016.49.10.071

Received 20 April 2016, Revised 23 May 2016, Accepted 15 July 2016

Keywords: Chondrocyte hypertrophy, Chondrogenesis, Cordycepin, Mesenchymal stem cells engineering techniquehas provided novel ways to repair cartilage defects (5). Mesenchymal stem cells (MSCs) have the potential of differentiation into chondrocytes. They are considered as appropriate seed cells in constructing cartilage through tissue engineering (6). The phenotype of chondrocyte derived from MSCs is difficult to maintain in cartilage reconstruction (7). Repair cells often lose chondrogenic activity which results in chondrocyte hypertrophy that can affect the formation of stable hyaline cartilage. Therefore, inhibiting chondrocyte hypertrophy in engineered cartilage is an enormous challenge (8).

Cordycepin, a nucleoside adenosine, is an effective substance of cordyceps (9). It has been reported that cordycepin possesses various biological functions, including antioxidant, hypolipidemic, and anti-inflammatory activities $(10,11)$. A recent study has confirmed that cordycepin may induce leydig tumor cells apoptosis via MAPK/P38 signaling (12). However, the role of cordycepin in cartilage reconstruction, especially in chondrocyte hypertrophy, remains unclear. Therefore, the objective of this study was to determine the effect of cordycepin on chondrogenesis and chondrocyte hypertrophy in MSCs and ATDC5 cells. Specifically, we determined whether cordycepin could promote chondrogenesis of MSCs. We also determined whether cordycepin could affect chondrocyte hypertrophy of MSCs and murine chondroprogenitor cell line ATDC5 cells at early stage and late stage (13). Additional, several crucial genes of signaling pathway and transcription factors were examined to explore the molecular mechanisms involved in the effect of cordycepin on chondrocyte hypertrophy.

\section{RESULTS}

Cordycepin promotes chondrogenesis of MSCs

MSCs were cultured with basal culture medium for 24,48 , or $72 \mathrm{~h}$ and treated with different concentrations of cordycepin followed by CCK8 assays. Results showed that the concentration of cordycepin lower than $1 \mu \mathrm{g} / \mathrm{ml}$ had slight effect $(\mathrm{P}>0.05)$ on cell proliferation (Fig. S1A, S1C, S1E). Therefore, cordycepinat concentration of $1 \mu \mathrm{g} / \mathrm{ml}$ was regarded as safe and used in this study. After induction by chondrogenic 
medium containing TGF- $\beta 3$ together with cordycepin for $14 \mathrm{~d}$, compared to control group Toluidine blue staining intensity was increased $(\mathrm{P}<0.05)$ following cordycepin treatment on day 14 (Fig. S2C). Quantitative PCR (qPCR) analysis revealed that the mRNA levels of chondrogenesis markers including Sox9, Col II, COMP, and Acan in MSCs treated with cordycepin were significantly increased $(\mathrm{P}<0.05)$ compared to group without cordycepin (Fig. 1D, S2B). After treatment with cordycepin, Sox9 protein level was increased on day 14 and Col II expression was increased on day 7 and 14 (Fig. 1B, S2E). Immunohistochemistry results also revealed that the expression levels of Sox9 and Col II were increased on day 7 and 14 based on the increases of integrated optical densities (Fig. 1C, S2A and S2D). Our data demonstrated that cordycepin promoted chondrogenesis at concentration of $1 \mu \mathrm{g} / \mathrm{ml}$.

\section{Cordycepin inhibits chondrocyte hypertrophy of MSCs at late stage}

After induction with chondrogenic differentiation medium containing TGF- $\beta 3$ for $14 \mathrm{~d}$ without cordycepin, MSCs were induced by hypertrophic medium containing T3 with cordycepin for another $14 \mathrm{~d}$ to examine the effect of cordycepin on chondrocyte hypertrophy. Cordycepinwas used at concentration of $1 \mu \mathrm{g} / \mathrm{ml}$. Toluidine blue staining showed that groups treated with cordycepin had higher $(P<0.05)$ intensity than control groups (Fig. 2A, 2C). Results of qPCR revealed, after cordycepin treatment, the expression levels of hypertrophic marker $\mathrm{Col} X$ in groups treated with cordycepin were increased $(\mathrm{P}<0.05)$ on day 17 and 21, MMP13levels were increased $(\mathrm{P}<0.05)$ only on day 17 , Runx2 levels were increased $(\mathrm{P}<$ $0.05)$ only on day 21, whereas the expression levels of MMP13 and Runx2 were decreased $(P<0.05)$ on day 28 compared with those in the control groups (Fig. 2D). Western blot analysis revealed that the protein levels of Col $\mathrm{X}$ and Runx2 in groups treated with cordycepin were not changed on
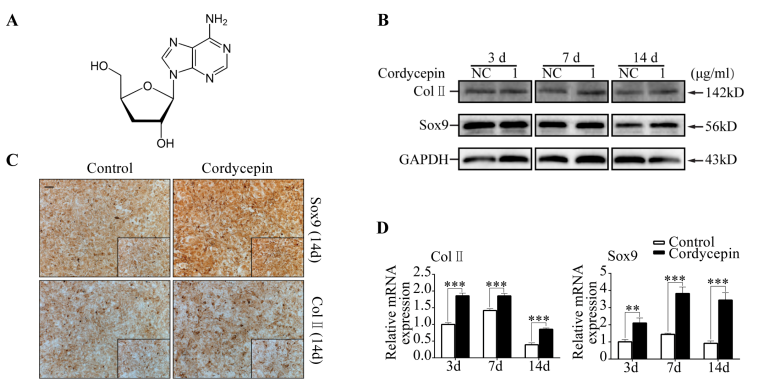

Fig. 1. Cordycepin promotes chondrogenesis of MSCs. MSCs were induced by chondrogenic medium containing TGF- $\beta 3$ for 3,7 , and $14 \mathrm{~d}$ with or without cordycepin. (A) Chemical formula of cordycepin. (B) Representative western blot images of Sox9, Col II, and GAPDH from MSCs in different groups (day 3, 7, and 14). (C) Representative immunohistochemical staining images of Sox9 and Col II from MSCs in different groups (day 14). Scale bar: 200 $\mu \mathrm{m}$. (D) Relative mRNA expression levels of Sox9 and Col II. day 17 but decreased on day 21 and 28 compared to those in the control groups (Fig. 2B, S3B). Immunohistochemistry results revealed that the expression levels of Col X and Runx2 in groups treated with cordycepin on day 21 and 28 were lower than those in the control groups based on integrated optical densities (Fig. S3A, S3C). These results demonstrated that cordycepin promoted chondrocyte hypertrophy of MSCs at early stage but inhibited chondrocyte hypertrophy of MSCs at late stage.

\section{Cordycepin inhibits chondrocyte hypertrophy of ATDC5 cells at late stage}

CCK8 assay was performed to examine the toxicity of cordycepin to ATDC5 cells. Concentration of cordycepin lower than $1 \mu \mathrm{g} / \mathrm{ml}$ had no significant $(\mathrm{P}>0.05)$ effect on cell viability (Fig. S1B, S1D, and S1F). Therefore, cordycepin was used at $1 \mu \mathrm{g} / \mathrm{ml}$ for the following experiments to avoid toxicity issue. Previous results showed that cordycepin inhibited MSCs chondrocyte hypertrophy. As a useful tool to study the differentiation of chondrocytes, ATDC5 cells were induced by hypertrophic medium containing T3 with cordycepin for $14 \mathrm{~d}$ to examine the effect of cordycepin on chondrocyte hypertrophy. After treatment with cordycepin, compared to control group Toluidine blue staining intensity was increased $(\mathrm{P}<$ 0.05) on day 14 (Fig. S4A). Results of qPCR revealed that the mRNA levels of hypertrophic markers including MMP13 and Runx2 were increased $(\mathrm{P}<0.05)$ in cells treated with cordycepin on day 3 but decreased $(P<0.05)$ on day 7 and 14 compared to group without cordycepin treatment (Fig. S4D). After treatment with cordycepin, Runx2 protein level was not changed $(P>0.05)$ on day 3 but decreased $(P<0.05)$ on day 7 and 14 compared to group without cordycepin treatment. $\mathrm{Col} X$ protein levels in cells treated with cordycepinwere increased $(P<0.05)$ on day 3 but decreased $(P<0.05)$ on day 14 compared to group without cordycepin treatment (Fig. $\mathrm{S} 4 \mathrm{E}, \mathrm{S} 4 \mathrm{~F})$. Immunohistochemistry results revealed that the
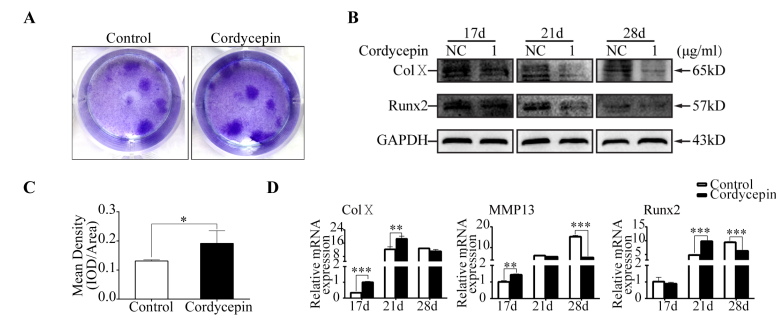

Fig. 2. Cordycepin inhibits chondrocyte hypertrophy of MSCs. MSCs were induced with chondrogenic medium for $14 \mathrm{~d}$ and then treated with hypertrophic medium for another 3, 7, and $14 \mathrm{~d}$. (A) Representative Toluidine blue staining images of MSCs in different groups (day 28). (B) Representative western blot images of Col X, Runx2, and GAPDH from MSCs in different groups (day 17, 21, and 28). (C) Quantification of mean intensity of toluidine blue staining. (D) Relative mRNA expression levels of Col X, MMP13, and Runx2. 
expression levels of $\mathrm{Col} X$ and Runx2in cells treated with cordycepin on day 7 and 14 were lower than those in control groups without cordycepin treatment based on integrated optical densities (Fig. S4B, S4C). Taken together, these results demonstrated that ATDC5 cells hypertrophy were promoted by cordycepin at early stage but inhibited at late stage.

\section{Cordycepin up-regulates BMP signaling during chondrogenesis and down-regulates Notch signaling during hypertrophy differentiation}

To explore the molecular mechanisms involved in the effect of cordycepinonTGF- $\beta 3$ induced chondrogenesis of MSCs, we performed qPCR and determined the expression levels of transcriptional factors and key molecules in canonical pathways related to chondrogenesis. After treatment with cordycepin, Hey 1 expression was increased on day 3 and 14 compared to that in control groups. Expression levels of Id 1 and $\mathrm{Id} 3$ were not changed by the treatment of cordycepin. However, Id2 expression was increased in treatment groups on day 14 compared to that in control groups. Moreover, the expression of $\mathrm{PI} 3 \mathrm{~K}$ was increased in the presence of cordycepin on day 3 and 7 whereas Nrf2 expression was decreased following cordycepin treatment on day 3, 7, and 14 (Fig. 3A). These results demonstrated that cordycepin promoted MSCs chondrogenesis through activating BMP and Notch signaling pathways. Besides, cordycepin up-regulated $\mathrm{PI} 3 \mathrm{~K}$ expression but down-regulated Nrf2 expression to facilitate MSCs chondrogenesis.

To further explore the molecular mechanisms, we performed qPCR to screen the expression of transcriptional factors and key molecules in canonical pathways associated with chondrocyte hypertrophy. In MSCs groups treated with cordycepin, the expression of $\beta$-catenin was increased on day 17 but

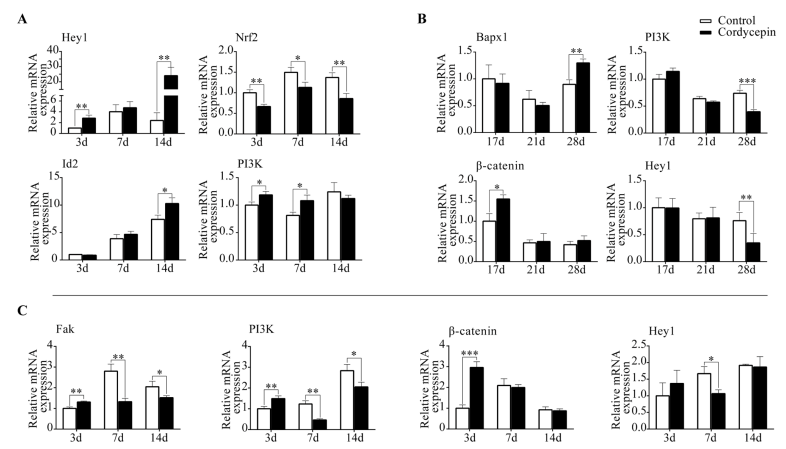

Fig. 3. Cordycepin promoteschondrogenesis through BMP pathway but inhibits chondrocyte hypertrophy through Notch pathway. (A) Relative mRNA expression levels of Hey1, Nrf2, Id2, and PI3K from MSCs induced by chondrogenic medium (day 3, 7, and 14). (B) Relative mRNA expression levels of Bapx1, PI3K, $\beta$-catenin, and Hey1 from MSCs in different groups (day 17, 21, and 28). (C) Relative mRNA expression levels of Fak, PI3K, $\beta$-catenin, and Hey1 from ATDC5 cells induced by hypertrophic medium in different groups (day 3, 7, and 14). unchanged on day 21 and day 28. Bapx 1 expression was increased on day 28. On the contrary, the expression of PI3K was decreased on day 28. The expression levels of Hey 1 and Hes5 (downstream genes in Notch signaling) were decreased on day 28. Hes 1 expression was decreased on both day 21 and day 28 compared to that in the control groups (Fig. 3B, $\mathrm{S} 5 \mathrm{~A})$. These results were similar to those obtained in ATDC5 cells. After treatment with cordycepin, the expression level of $\beta$-catenin was increased on day 3 but unchanged on day 7 or 14 compared to that in the control groups. Besides, after cordycepin treatment, Hes1 expression was decreased on day 3,7 , and 14, Hes5 expression was decreased on day 7 and 14 , and Hey 1 expression was decreased only on day 7 . The expression of FAK (a positive regulator of chondrocyte hypertrophy) was increased following cordycepin treatment on day 3 but decreased on day 7 and 14. PI3K was the downstream signaling molecule of FAK. Its expression change was consistent with that of FAK (Fig. 3C, S5B). Taken together, these results indicated that cordycepin up-regulated BMP signaling during chondrogenesis but down-regulated Notch signaling during hypertrophy differentiation.

\section{DISCUSSION}

Cordycepin has been considered as an effective antioxidative agent with protective effects on respiratory system (14), nervous system (15), endocrine system (16), and tumor metastasis (12, $17,18)$. Based on the characteristics of cordycepin, we hypothesized that cordycepin might also have effect on cartilage metabolism. A previous study has shown that cordycepin has protection against inflammatory cytokine inhibition of osteogenic differentiation of human adipose-derived mesenchymal stem cells (19). A recent study has also demonstrated that cordycepin could prevent oxidative stress-induced inhibition of osteogenesis (20).

In this study, we revealed that cordycepin had positive effect on TGF- $\beta 3$ induced chondrogenesis of MSCs. Several specific genes related to cartilage such as Sox9 and Col II $(21,22)$ were examined in this study to explain the phenomenon at molecular level. These genes were up-regulated by cordycepin treatment. We then focused on two important signaling pathways during chondrogenesis: Notch and BMP. Notch signaling in MSCs is necessary for the initiation of chondrogenesis $(23,24)$. Besides, inhibitor of DNA binding/differentiation 1 (Id1), Id2, and Id3 are involved in the BMP signaling pathway indispensable for the formation of cartilage following the activation of the Col II gene (25-27). Our qPCR results showed that cordycepin could promote Notch signaling pathway during chondrogenesis. However, cordycepin started to show positive effect on BMP signaling pathway on day 14 . In other words, BMP signaling was involved in the regulation of cordycepin at late stages of chondrogenic differentiation. During differentiation of MSCs into chondrocytes, the activation of $\mathrm{PI} 3 \mathrm{~K}$ can positively regulate chondrogenesis (28-30). Nrf2 negatively regulates cellular 
differentiation toward maturation in chondrocytes (31). We found that cordycepin could facilitate MSCs chondrogenesis by up-regulating PI3K expression while down-regulating Nrf2 expression.

As an important transcription factor in chondrocyte hypertrophy, Runx2 promotes the expressions of MMP13 and Col X, thus disturbing chondrocyte homeostasis. Cordycepin had dual effects on chondrocyte hypertrophy both in MSCs and ATDC5 cells. Expression levels of specific genes related to chondrocyte hypertrophy including Runx2, MMP13, and Col $X$ were tested in this study and results consistently revealed that cordycepin had dual effects. Interestingly, we found that the protein levels of Runx2 and Col X in MSCs were inhibited by cordycepin starting from $21 \mathrm{~d}$ while their mRNA levels were inhibited on day 28. Wnt signaling and Notch signaling are reported to have positive effect on chondrocyte hypertrophy during chondrogenesis $(32,33)$. Bapx 1 can suppress chondrocyte hypertrophy in ATDC5 cells (34). PI3K-mediated suppression of Bapx 1 in chondrocytes also plays a role in the control of cartilage hypertrophy during skeletal development in vertebrates (35). It is well known that FAK activates multiple integrin-mediated signal transduction pathways such as PI3K/Akt pathway (36). The expression of FAK is reported to be significantly higher in osteoarthritis chondrocytes compared to normal control group (37). Therefore, FAK could be a positive regulator of chondrocyte hypertrophy. In MSCs, cordycepin promoted chondrocyte hypertrophy through Wnt signaling and inhibited chondrocyte hypertrophy through Notch signaling. The expression of PI3K was decreased following cordycepin treatment on day 28 whereas the expression of Bapx 1 was increased. Therefore, cordycepin reduced the inhibitory effect of PI3K to Bapx1, thus inhibiting chondrocyte hypertraophy at late stage. As for ATDC5 cells, Wnt and Notch signaling pathways were involved in the regulation of cordycepin at early stage and late stage of chondrocyte hypertrophy, respectively. With cordycepin treatment, the expression of FAK and PI3K were increased at early stage of chondrocyte hypertrophy but decreased at late stage of chondrocyte

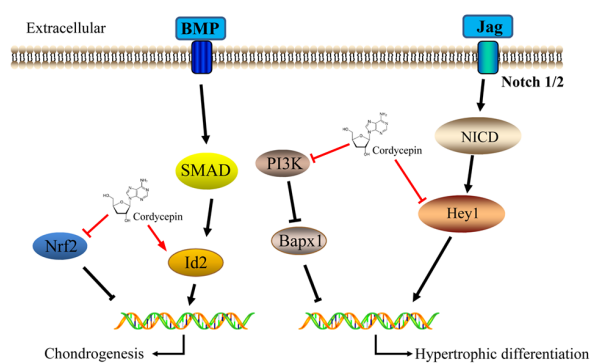

Fig. 4. Schematic diagram of cordycepin function in chondrogenesis and chondrocyte hypertrophy of MSCs. Cordycepin promotes chondrogenesis through inhibiting Nrf2 and activating BMP signaling. Besides, cordycepin suppresses chondrocyte hypertrophy through PI3K/Bapx1 pathway and Notch signaling. hypertrophy. Hence, cordycepin regulated the promoting role of FAK on PI3K and inhibited chondrocyte hypertrophy.

Chondrocyte homeostasis is critical for the stability of tissue engineered cartilage (38). The effect of cordycepin on chondrogenesis and chondrocyte hypertrophy of MSCs might provide us new insights (Fig. 4). Due to its promoting effect on chondrogenesis, cordycepin can facilitate seed cells differentiation towards chondrocytes to accelerate cartilage repair. In addition, cordycepin can reduce degradation of cartilage matrix to balance anabolic and catabolic cellular activity of repair cells. These data indicate that cordycepin might have potential clinical application to modulate cartilage homeostasis and reconstruct cartilage.

\section{MATERIALS AND METHODS}

\section{Cell culture}

C3H10T1/2 mesenchymal stem cells were purchased from the American Type Culture Collection (ATCC, Manassas, VA, USA). ATDC5 cells were obtained from RIKEN cell Bank (Tsukuba, Japan). These cells were cultured in DMEM-F12 (Gibco Life Technology, Gaithersburg, MD, USA) containing $10 \%$ fetal bovine serum (FBS) (Gibco) and 1\% penicillinstreptomycin solution (Beyotime Biotechnology, Shanghai, China) at $37^{\circ} \mathrm{C}$ with $5 \% \mathrm{CO} 2$. The culture medium was replaced every 2-3 d until cells reached $90 \%$ confluence. Cells were passaged after dissociating cells with $0.25 \%$ trypsin (Gibco) at room temperature for $2 \mathrm{~min}$. The second passage cells were used for the following experiments.

\section{In vitro cell proliferation assay}

C3H10T1/2 cells and ATDC5 cells were seeded $\left(2 \times 10^{3}\right.$ cells per well) into 96-well plates in triplicates and allowed to adhere overnight. On the following day, the medium was replaced by fresh medium containing cordycepin (Sigma-Aldrich Company). Cultures were incubated for 24,48 , or 72 hours. To determine cell viabilities, $10-\mu$ l of Cell Counting Kit-8 (CCK8, Dojindo Laboratories) reagent was added to each well and incubated for an additional 4 hours. The absorbance was read at wavelength of $450 \mathrm{~nm}$ on an automated plate reader. Wells containing CCK8 reagent without cells were used as blank controls. Cell proliferation was assessed based on the absorbance values according to the manufacturer's protocol.

\section{Chondrogenic differentiation assay}

C3H10T1/2 cells were dissociated using $0.25 \%$ trypsin and adjusted to a density of $10^{5}$ cells $/ \mathrm{ml}$. Cell suspension (2 $\mathrm{ml} /$ well) was placed into the center of each well on a 6-well plate. After incubation for $24 \mathrm{~h}$ at $37^{\circ} \mathrm{C}$ with $5 \% \mathrm{CO}_{2}$, the medium was replaced by $2 \mathrm{ml}$ chondrogenic differentiation medium (Gibco) containing dexamethasone (100 nmol/L), ascorbate $(50 \mu \mathrm{g} / \mathrm{ml})$, ITS + Supplement, proline $(40 \mu \mathrm{g} / \mathrm{ml})$, and TGF- $\beta 3(10 \mathrm{ng} / \mathrm{ml})$. The chondrogenic differentiation medium was refreshed every $3 \mathrm{~d}$. 


\section{Hypertrophic differentiation assay}

ATDC5 cells were dissociated with $0.25 \%$ trypsin and adjusted to a density of $10^{5}$ cell $\mathrm{s} / \mathrm{ml}$. Cell suspension was placed into the center of each well on a 6-well plate (2 $\mathrm{ml} /$ well). After incubation at $37^{\circ} \mathrm{C}$ with $5 \% \mathrm{CO}_{2}$ for $24 \mathrm{~h}$, the medium was replaced by $2 \mathrm{ml}$ hypertrophic differentiation medium (Gibco) containing dexamethasone (1 nmol/L), ascorbate $(50 \mu \mathrm{g} / \mathrm{ml})$, ITS + Supplement, proline $(40 \mu \mathrm{g} / \mathrm{ml})$, and triiodothyronine $(\mathrm{T} 3,100 \mathrm{ng} / \mathrm{ml})$. The hypertrophic differentiation medium was refreshed every $3 \mathrm{~d}$.

\section{Quantitative PCR (qPCR) analysis}

Total RNA was isolated from cells using Trizol reagent (Life Technologies). Single-stranded cDNA was prepared from $1 \mu \mathrm{g}$ of total RNA using reverse transcriptase and oligo-dT primer using First Strand cDNA synthesis kit (TaKaRa Biotechnology Co.) according the manufacturer's instructions. Each cDNA (2 $\mu$ ) was subjected to qPCR amplification using gene specific primers (Table S1). The cycling conditions were set as $95^{\circ} \mathrm{C}$ for 30 seconds, 40 cycles of $95^{\circ} \mathrm{C}$ for 5 seconds, and $60^{\circ} \mathrm{C}$ for 30 seconds. The relative mRNA level was calculated by the normalization to that of GAPDH (39).

\section{Western blot analysis}

Cells were extracted with cell lysis buffer [50 mM Tris $(\mathrm{pH}$ 7.6), $150 \mathrm{mM} \mathrm{NaCl}, 1 \%$ TritonX-100, $1 \%$ deoxycholate, $0.1 \%$ SDS, $1 \mathrm{mM}$ PMSF, and 0.2\% Aprotinin (Beyotime)]. After measuring protein concentration by BCA protein assay (Beyotime), equal protein amounts were mixed with $5 \times$ sample buffer (Beyotime) and boiled. These samples were resolved on $10 \%$ SDS-PAGE (sodium dodecyl sulfate polyacrylamide gel electrophoresis) and transferred onto PVDF (polyvinylidene fluoride) membrane by using semi-dry transfer method. After blocking in $10 \%$ nonfat dry milk in TBST (tris-buffered saline tween-20) for $2 \mathrm{~h}$, blots were incubated with primary antibodies including Col II (rabbit polyclonal 1:500, Abcam), Sox9 (rabbit polyclonal 1:500, Abcam), Runx2 (rabbit polyclonal 1:500, Abcam), Col X (rabbit polyclonal 1:500, Abcam) and GAPDH (rabbit polyclonal 1:1,000, Abcam) at $4^{\circ} \mathrm{C}$ overnight. After washing with TBST three times, blots were incubated with goat anti-rabbit secondary antibody $(1: 2,000$, ZSGB-BIO) at room temperature for $1 \mathrm{~h}$. GAPDH was used as loading control.

\section{Glycosaminoglycan (GAG) synthesis analysis by Toluidine blue staining \\ To demonstrate the deposition of cartilage matrix proteoglycans, representative cultures were collected at indicated time points (day 7,14 , and 28) of induction and sulfated cartilage glycosa- minoglycans (GAGs) were measured by Toluidine blue (Beyotime) staining. Cells were fixed in $4 \%$ paraformaldehyde for $15 \mathrm{~min}$ and stained with Toluidine blue for $5 \mathrm{~min}$. The mean density was normalized to total cell number.}

\section{Immunohistochemistry}

Cells were fixed in $4 \%$ paraformaldehyde for $15 \mathrm{~min}$, washed in phosphate-buffered saline (PBS), and treated with $\mathrm{H}_{2} \mathrm{O}_{2}$ (ZSGB-BIO Biotechnology, Beijing, China) for $10 \mathrm{~min}$ to inactivate endogenous peroxidase. After treatment with normal goat serum (ZSGB-BIO) at room temperature for $15 \mathrm{~min}$, cells were incubated with primary antibody including Col II (rabbit polyclonal 1:500, Abcam), Sox9 (rabbit polyclonal 1:500, Abcam), Runx2 (rabbit polyclonal 1:500, Abcam) and Col X (rabbit polyclonal 1:500, Abcam) at $4^{\circ} \mathrm{C}$ overnight. After washing with PBS, cells were incubated with biotinylated goat anti-rabbit (ZSGB-BIO) secondary antibodies for $30 \mathrm{~min}$ followed by washing and incubation with horseradish peroxidase (HRP) conjugated secondary antibody (ZSGB-BIO) for $15 \mathrm{~min}$. The area of the immunocomplex was visualized using chromogen 3, 3'-diaminobenzidine (DAB) as substrate for $5 \mathrm{~min}$. Cells were observed under Olympus fluoresence microscope (IX71). Image-Pro plus 6.0 software was used for image analysis.

\section{Statistics}

Statistical significance was determined using SPSS 13.0 and data were presented as mean \pm standard deviation. ${ }^{*} \mathrm{P}<0.05$, $* * \mathrm{P}<0.01$, and $* * * \mathrm{P}<0.001$ were regarded as statistically significant.

\section{ACKNOWLEDGEMENTS}

This work was funded by grants from the Nature Science Foundation of China (81271980, 81571893), the National High-tech R\&D Program of China (863 Program, 2015AA 020315), and the National Key Technology Research and Development Program of China (2012BAI42G01).

\section{REFERENCES}

1. Madry $\mathrm{H}$ and Cucchiarini M (2014) Tissue-engineering strategies to repair joint tissue in osteoarthritis: nonviral gene-transfer approaches. Curr Rheumatol Rep 16, 450

2. Mardones R, Jofre CM and Minguell JJ (2015) Cell Therapy and Tissue Engineering Approaches for Cartilage Repair and/or Regeneration. Int J Stem Cells 8, 48-53

3. Caldwell KL and Wang J (2015) Cell-based articular cartilage repair: the link between development and regeneration. Osteoarthritis Cartilage 23, 351-362

4. He B, Yuan X, Zhou A, Zhang H and Jiang D (2014) Designer functionalised self-assembling peptide nanofibre scaffolds for cartilage tissue engineering. Expert Rev Mol Med 16, e12

5. Sun AX, Lin H, Beck AM, Kilroy EJ and Tuan RS (2015) Projection Stereolithographic Fabrication of Human Adipose Stem Cell-Incorporated Biodegradable Scaffolds for Cartilage Tissue Engineering. Front Bioeng Biotechnol 3, 115

6. Bradamante S, Barenghi L and Maier JA (2014) Stem Cells toward the Future: The Space Challenge. Life (Basel) 4, 
267-280

7. Kaul G, Cucchiarini M, Remberger K, Kohn D and Madry H (2012) Failed cartilage repair for early osteoarthritis defects: a biochemical, histological and immunohistochemical analysis of the repair tissue after treatment with marrow-stimulation techniques. Knee Surg Sports Traumatol Arthrosc 20, 2315-2324

8. Sun MM and Beier F (2014) Chondrocyte hypertrophy in skeletal development, growth, and disease. Birth Defects Res C Embryo Today 102, 74-82

9. Nakamura K, Shinozuka K and Yoshikawa N (2015) Anticancer and antimetastatic effects of cordycepin, an active component of Cordyceps sinensis. J Pharmacol Sci $127,53-56$

10. Tuli HS, Sharma AK, Sandhu SS and Kashyap D (2013) Cordycepin: a bioactive metabolite with therapeutic potential. Life Sci 93, 863-869

11. Yue K, Ye M, Zhou Z, Sun W and Lin X (2013) The genus Cordyceps: a chemical and pharmacological review. J Pharm Pharmacol 65, 474-493

12. Pan BS, Wang YK, Lai MS, Mu YF and Huang BM (2015) Cordycepin induced MA-10 mouse Leydig tumor cell apoptosis by regulating p38 MAPKs and PI3K/AKT signaling pathways. Sci Rep 5, 13372

13. Yao $Y$ and Wang Y (2013) ATDC5: an excellent in vitro model cell line for skeletal development. J Cell Biochem $114,1223-1229$

14. Yang X, Li Y, He Y et al (2015) Cordycepin alleviates airway hyperreactivity in a murine model of asthma by attenuating the inflammatory process. Int Immunopharmacol 26, 401-408

15. Peng J, Wang $P, G e ~ H, Q u X$ and Jin X (2015) Effects of cordycepin on the microglia-overactivation-induced impairments of growth and development of hippocampal cultured neurons. PLoS One 10, e0125902

16. Ma L, Zhang S and Du M (2015) Cordycepin from Cordyceps militaris prevents hyperglycemia in alloxaninduced diabetic mice. Nutr Res 35, 431-439

17. Kang FC, Chen PJ, Pan BS, Lai MS, Chen YC and Huang BM (2015) Apoptotic effect of cordycepin combined with cisplatin and/or paclitaxel on MA-10 mouse Leydig tumor cells. Onco Targets Ther 8, 2345-2360

18. Li Y, Li R, Zhu S et al (2015) Cordycepin induces apoptosis and autophagy in human neuroblastoma SK-N-SH and BE(2)-M17 cells. Oncol Lett 9, 2541-2547

19. Yang J, Cao Y, Lv Z, Jiang T, Wang L and Li Z (2015) Cordycepin protected against the TNF-alpha-induced inhibition of osteogenic differentiation of human adiposederived mesenchymal stem cells. Int J Immunopathol Pharmacol 28, 296-307

20. Wang F, Yin P, Lu Y et al (2015) Cordycepin prevents oxidative stress-induced inhibition of osteogenesis. Oncotarget 6, 35496-35508

21. Healy C, Uwanogho D and Sharpe PT (1999) Regulation and role of Sox9 in cartilage formation. Dev Dyn 215, 69-78

22. Wu W, Mwale F, Tchetina E, Kojima T, Yasuda T and Poole AR (2001) Cartilage matrix resorption in skeletogenesis. Novartis Found Symp 232, 158-166; discussion $166-170$
23. Oldershaw RA and Hardingham TE (2010) Notch signaling during chondrogenesis of human bone marrow stem cells. Bone 46, 286-293

24. Ma Y, Bian J and Zhang F (2015) Inhibition of perillyl alcohol on cell invasion and migration depends on the Notch signaling pathway in hepatoma cells. Mol Cell Biochem 411, 307-315

25. Karystinou A, Roelofs AJ, Neve A, Cantatore FP, Wackerhage $\mathrm{H}$ and De Bari C (2015) Yes-associated protein (YAP) is a negative regulator of chondrogenesis in mesenchymal stem cells. Arthritis Res Ther 17, 147

26. Long F and Ornitz DM (2013) Development of the endochondral skeleton. Cold Spring Harb Perspect Biol 5, a008334

27. Dong J, Lei X, Wang Y et al (2015) Different Degrees of Iodine Deficiency Inhibit Differentiation of Cerebellar Granular Cells in Rat Offspring, via BMP-Smad1/5/8 Signaling. Mol Neurobiol 53, 4606-4617

28. Shang J, Liu H, Li J and Zhou Y (2014) Roles of hypoxia during the chondrogenic differentiation of mesenchymal stem cells. Curr Stem Cell Res Ther 9, 141-147

29. Mitsugi S, Ariyoshi W, Okinaga T et al (2012) Mechanisms involved in inhibition of chondrogenesis by activin-A. Biochem Biophys Res Commun 420, 380-384

30. Li Z, Lu H and Li ZB (2014) The PI3K/Akt signalling pathway may play an internal role related to abnormal condylar growth: a preliminary study. Int J Oral Maxillofac Surg 43, 1477-1483

31. Hinoi E, Takarada T, Fujimori S et al (2007) Nuclear factor E2 p45-related factor 2 negatively regulates chondrogenesis. Bone 40, 337-344

32. Choi SW, Jeong DU, Kim JA et al (2012) Indian Hedgehog signalling triggers $\mathrm{Nkx3.2}$ protein degradation during chondrocyte maturation. Biochem J 443, 789-798

33. Kohn A, Dong Y, Mirando AJ et al (2012) Cartilage-specific RBPjkappa-dependent and -independent Notch signals regulate cartilage and bone development. Development 139, 1198-1212

34. Caron MM, Emans PJ, Cremers A et al (2013) Hypertrophic differentiation during chondrogenic differentiation of progenitor cells is stimulated by BMP-2 but suppressed by BMP-7. Osteoarthritis Cartilage 21, 604-613

35. Kim JA, Im S, Cantley LC and Kim DW (2015) Suppression of $\mathrm{Nkx3.2}$ by phosphatidylinositol-3-kinase signaling regulates cartilage development by modulating chondrocyte hypertrophy. Cell Signal 27, 2389-2400

36. Thamilselvan V, Craig DH and Basson MD (2007) FAK association with multiple signal proteins mediates pressure-induced colon cancer cell adhesion via a Src-dependent PI3K/Akt pathway. FASEB J 21, 1730-1741

37. Cheng K, Xia P, Lin Q et al (2014) Effects of low-intensity pulsed ultrasound on integrin-FAK-PI3K/Akt mechanochemical transduction in rabbit osteoarthritis chondrocytes. Ultrasound Med Biol 40, 1609-1618

38. Chen S, Fu P, Cong R, Wu H and Pei M (2015) Strategies to minimize hypertrophy in cartilage engineering and regeneration. Genes Dis 2, 76-95

39. Bustin SA, Benes V, Nolan T and Pfaffl MW (2005) Quantitative real-time RT-PCR-a perspective. J Mol Endocrinol 34, 597-601 